\title{
Towards a Semantic Web Platform for Finite Element Simulations
}

\author{
André Freitas ${ }^{1}$, Kartik Asooja ${ }^{1}$, Swapnil Soni ${ }^{1,2}$, Marggie Jones ${ }^{1}$, Panagiotis \\ Hasapis $^{3}$, Ratnesh Sahay ${ }^{1}$ \\ ${ }^{1}$ Insight Centre for Data Analytics, National University of Ireland, Galway \\ ${ }^{2}$ Kno.e.sis Center, Wright State University \\ ${ }^{3}$ Intrasoft International
}

\begin{abstract}
Finite Element (FE) simulations are present in many different branches of science. The growth in the complexity of FE models and their associated costs bring the demand to facilitate the construction, reuse and reproducibility of FE models. This work demonstrates how Semantic Web technologies can be used to represent FE simulation data, improving the reproducibility and automation of FE simulations.
\end{abstract}

\section{Motivation}

Scientific investigation practice is evolving in the direction of the creation of large-scale, highly complex, multi-domain and multi-scale scientific models and theories [1. The complexity intrinsic to these models, brings barriers for interpreting, reproducing and reusing third-party scientific results. The lack of reproducibility in science and the effort necessary for reusing and adapting existing scientific models are major problems in contemporary scientific praxis.

Finite Element (FE) methods are numerical techniques for finding approximate solutions for differential equations, and are examples of computational models which are present in different branches of science, including Biology, Physics and Engineering. The construction of FE models is a high complexity task which depends on multiple steps, including the definition of a discretized geometrical model (a mesh), the definition of a physical model, the selection of numerical methods, the visualization and interpretation of the results and the experimental validation of the model. Building a consistent and realistic FE model is an empirical and time consuming process, depending on the composition and fine-tuning of different parameters. This complexity is expressed in the difficulty of building and validating FE Models and in reproducing and reusing third-party FE models. Previous works in the area have concentrated on the representation of $\mathrm{FE}$ elements in the mechanical engineering design domain [2]. This work targets the FE modeling problem from an eScience perspective.

This work describes the SIFEMacronym for Semantic Infostructure for Finite Element Methods platform, a semantic infrastructure to support the construction, validation, reproducibility and reuse of Finite Element models. The next sections provide a brief description and demonstration of the SIFEM system. 


\section{Semantic Infrastructure}

The motivation for the construction of a semantic infrastructure for FE simulations is twofold: (i) facilitating the construction and validation of multi-scale FE models and (ii) increasing the reuse and reproducibility of scientific models based on FE simulations. Both motivations are dependent on a standards-based representation model for $\mathrm{FE}$ data. In the first case, existing experimental and simulation data can be used to validate input parameters for simulations. A FE model consists of the specification of a geometrical model, material properties, boundary conditions, a physical model, numerical methods and reference experimental data. A standards-based representation supports the collaborative construction of a reference FE knowledge base, which can be reused across different simulations, facilitating the validation of existing models against previous simulations and real world experimental data, and also the selective exploration of the simulation parameter space (by the elimination of unrealistic models previously explored). With the growth of the knowledge base, the exploration of the input parameter space can be partially automated, where domain experts specify a range of values and non-realistic or inconsistent parameters are automatically eliminated.

Additionally, the SIFEM platform allow users to specify data analysis rules, which support the semi-automated analysis of the results of different simulation scenarios. The simulation numerical results are lifted to a qualitative/symbolic level, through a feature extraction process. The features allow users to specify the expected behaviour of an output variable as a qualitative data analysis rule (Figure 1).

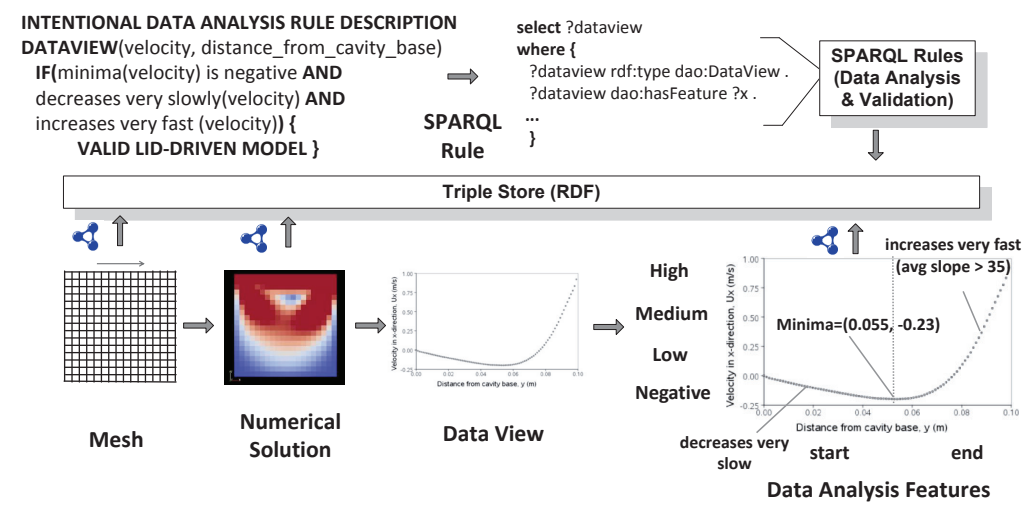

Fig. 1. Finite Element data at different steps in the simulation workflow.

In this work, the standardized Semantic Web data model $(\operatorname{RDF}(\mathrm{S}))$ and the reuse of existing vocabularies are used to maximize the interoperability of the 
generated simulation data. The standards-based representation also facilitates the composition of multi-scale FE models, i.e. models in which interacting but distinct physical systems at different scales are combined into a complex FE model. From the reproducibility perspective, a standardized data and conceptual model, in combination with the SIFEM web interface, allows the publication of simulation data and code artefacts directly linked from its paper description. While the combination of $\mathrm{RDF}(\mathrm{S})$ and vocabularies provides a well-known pattern for maximizing data interoperability, the main contribution of this work concentrates on the use of Semantic Web technologies in the automation of FE experiments.

Figure 2 shows the high-level components of the SIFEM platform and a typical usage workflow of the platform (represented by the number sequence). The workflow starts with the specification of the simulation parameters and of different simulation scenarios on the Simulation Manager component (1). This specification of the simulation is stored in the RDF triple store using a prospective provenance representation. The user then specifies the expected qualitative behavior of output variables through the Rule Creator component (2), using data analysis rules over data analysis features (Figure 1). After the specification of the simulation scenarios, the user starts the simulation using the Simulation Manager (3), which invokes the Execution Manager to coordinate the execution of the components of the simulation. The Execution Manager reads the solver input data from the RDF triple store and converts into the solver input format (Solver Configurator component) (4). The Execution Manager then invokes the solver for each simulation scenario, which generates the solver output (5). The Semantic Converter (6) component maps the Solver output data into the conceptual model, persisting it as RDF. Based on the specified data analysis rules, the Data Analyzer (7) component extracts a set of data analysis features from the simulation data on the triple store, and verifies if the specified data analysis rules are satisfied. The Data Analysis output is also persisted on the triple store. After the data is persisted, users can access the data using the Data Visualization and the SPARQL Endpoint components.

A FE simulation in SIFEM is represented using a multi-layered conceptual model. The conceptual model layers are depicted on Figure 2 and consist of the following domains: simulation, data analysis, finite element, physics, material properties and topology. Figure 2 also depicts the main ontologies which are reused and extended in the SIFEM system. Additional details on the SIFEM conceptual model can be found in 1 .

\section{Demonstration}

The demonstration 1 shows the use of the SIFEM platform for specifying, executing and analysing a set of FE simulations based on the lid-driven cavity flow (LDCF) model. The LDCF is a benchmark model for FE simulations and consists of box filled with a fluid with a moving lid. The user starts by uploading the

\footnotetext{
${ }^{1}$ The demonstration video can be found in http://bit.ly/1j4nVup
} 


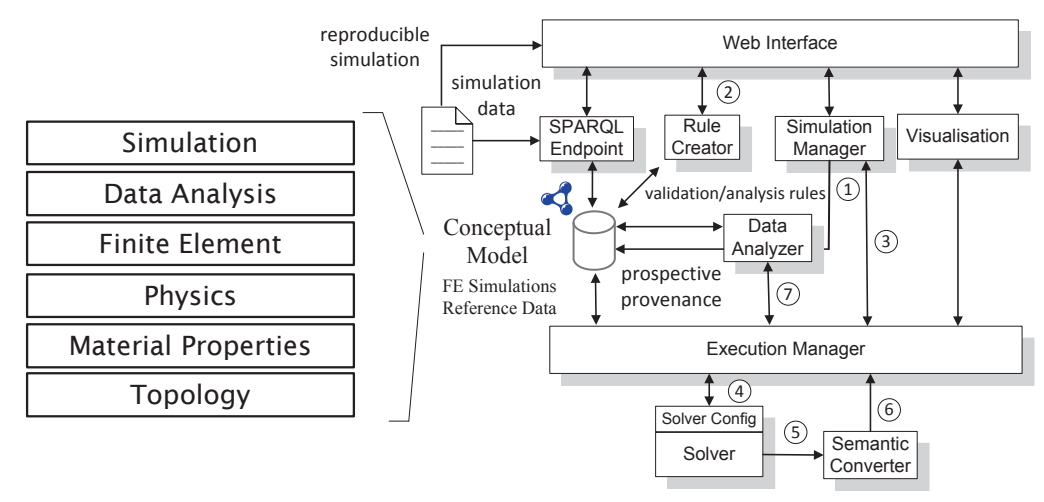

Fig. 2. High-level components of the SIFEM system and conceptual model.

geometric discretized model (mesh) into the platform. Based on the mesh model, the geometric patches are loaded and the user specifies the boundary conditions for different simulation scenarios. The user then specifies the material properties, the solver and the numerical method for the simulation. In the demonstration example, the user specified five different scenarios with varying lid velocities. Input parameters such as kinematic viscosity are validated against a reference model, by using validation rules (encoded as SPARQL Rules). The specification of the simulation scenarios is loaded into a triple store, following the conceptual model (Figure 2).

From the simulation specification, through the execution manager, the platform instantiates different simulation scenarios in the RDF triple store. The RDF simulation specification data for each scenario is loaded into the solver, which executes the simulation. For this example, the solver is solving the Navier-Stokes equation by using the PCG Finite Differences numerical method to calculate the fluid velocity and pressure at each mesh element. The solver outputs the velocity and pressure fields for each element in the mesh, at different points in time. After the simulation is finished and the data is loaded in the triple store, different data visualizations can be selected by users. In the example demonstration, the velocity and pressure fields over the mesh, and the graphs velocity $(x)$ vs. distance from cavity base and pressure vs. distance from cavity base are shown.

Different simulation scenarios from the experiment can be analysed by the specification of data analysis rules. This mechanism allows users to verify which simulation results match an expected output behavior. The demonstration uses the data analysis rule in Figure 1, In the demonstration, all the scenarios satisfy the rule, i.e. a vortex was generated above the center of the box.

The triple store data is accessible through a SPARQL Endpoint and as Linked Data through dereferenceable URIs. In the SPARQL Endpoint users can retrieve simulations with specific properties and compare different simulations. Examples of queries are: (i) What are the maximum fluid pressures for each scenario?, 
(ii) Which simulations are using the PCG numerical method? and (iii) Which kinematic viscosity values are used?

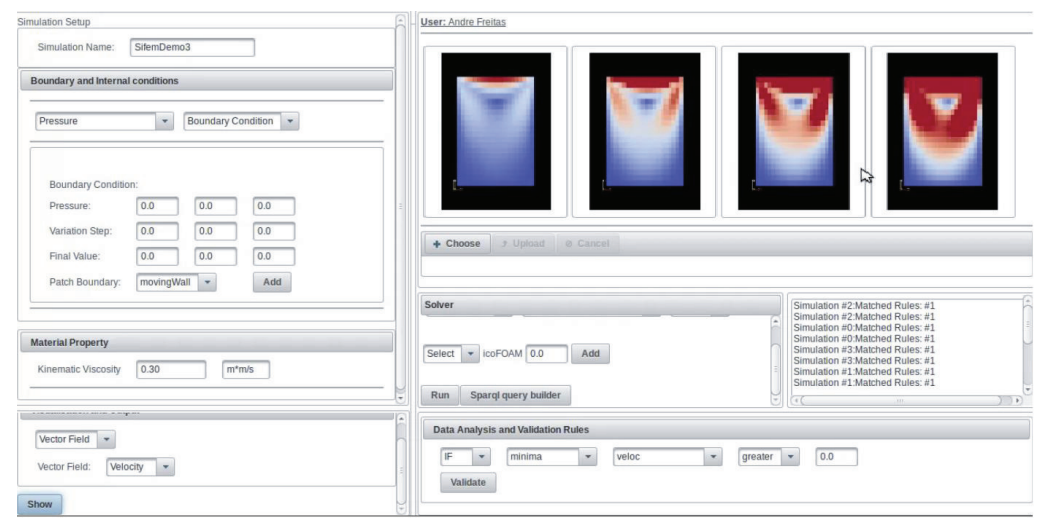

Fig. 3. Screenshot of the SIFEM Platform for the Lid-driven cavity flow simulation.

In the demonstration, the simulation and the associated Linked Data are directly linked from an example publication: a user clicks on a graph in an article and the associated simulation specification is opened on the SIFEM web interface. This allows the reader (who now becomes an interactive user) to re-run the simulations described in the paper, thereby supporting reproducibility and verifiability. The user is also enabled to run their own 'what if' scenarios using their choice of input parameters, facilitating further experimentation (possibly exploring scenarios not envisaged by the original model creators).

\section{Conclusions \& Future Work}

This work demonstrated the SIFEM platform, which uses Semantic Web technologies to increase the reproducibility, reusability and automation of Finite Element (FE) simulations. Future work includes the generalisation of the platform to cope with more complex FE simulations.

Acknowledgments. This work is supported by the EU: Grant No. 600933 (SIFEM).

\section{References}

1. Freitas et al., Towards a Semantic Representation for Multi-Scale Finite Element Biosimulation Experiments, 13th IEEE Intl. Conf. on BioInf. and BioEng., 2013.

2. Sun et al., A Framework for automated finite element analysis with an ontologybased approach, Journal of mechanical science and technology, 2009. 